\title{
Molecular Dynamics Simulations Identify Tractable Lead-like Phenyl-Piperazine Scaffolds as elF4A1 ATP-competitive Inhibitors
}

\author{
Derek J. Essegian, Tyler A. Cunningham, Christopher J. Zerio, Eli Chapman, Jonathan Schatz, \\ and Stephan C. Schürer*
}

Cite This: ACS Omega 2021, 6, 24432-24443

Read Online

ABSTRACT: eIF4A1 is an ATP-dependent RNA helicase whose overexpression and activity have been tightly linked to oncogenesis in a number of malignancies. An understanding of the complex kinetics and conformational changes of this translational enzyme is necessary to map out all targetable binding sites and develop novel, chemically tractable inhibitors. We herein present a comprehensive quantitative analysis of eIF4A1 conformational changes using protein-ligand docking, homology modeling, and extended molecular dynamics simulations. Through this, we report the discovery of a novel, biochemically active phenyl-piperazine pharmacophore, which is predicted to target the ATP-binding site and may serve as the starting point for medicinal chemistry optimization efforts. This is the first such report of an ATP-

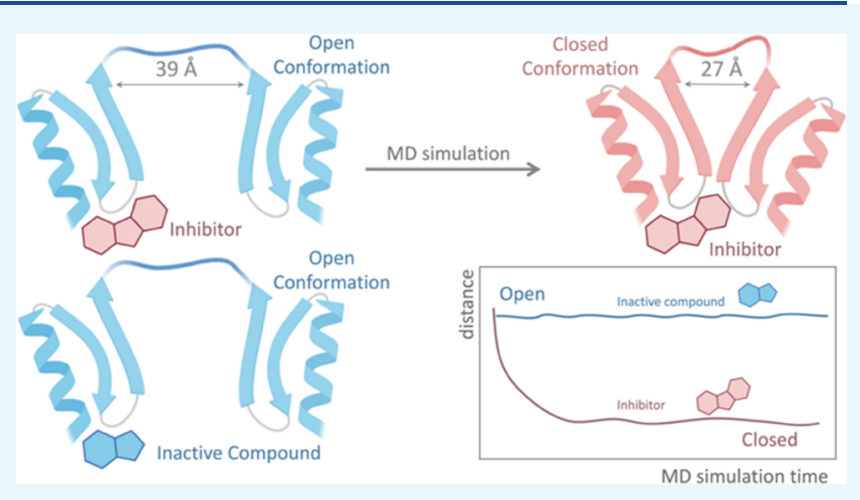
competitive inhibitor for eiF4A1, which is predicted to bind in the nucleotide cleft. Our novel interdisciplinary pipeline serves as a framework for future drug discovery efforts for targeting eiF4A1 and other proteins with complex kinetics.

\section{INTRODUCTION}

Dysregulation of protein translation is known to contribute to the establishment of many of the hallmarks of cancer, including evasion of apoptotic signals, altered cellular energetics and metabolism, invasion and metastasis, and induction of angiogenesis. ${ }^{1,2}$ In fact, many of the major pathways hijacked by cancer, such as $\mathrm{PI} 3 \mathrm{~K} / \mathrm{AKT} / \mathrm{mTOR}$ and $\mathrm{MEK} / \mathrm{Mnk}$, converge on the eukaryotic initiation factor (eIF) $4 \mathrm{~F}$ complex to promote cap-dependent translation of downstream oncogenic effector proteins. ${ }^{3,4}$ Overexpression of eIF4A1 in cancer has been correlated with both poor prognosis and drug resistance. ${ }^{5,6}$ Initial concern against globally targeting translation occluded interests in pursuing drug discovery and target validation studies of eIF4A1. However, it has since been established that there is a large therapeutic window in eIF4A1 inhibition, likely due to the aberrant upregulation in activity for protein translation in cancer cells compared to normal cells. ${ }^{7,8}$ While some highly potent and selective eIF4A1 inhibitors have been discovered, they lack core drug-like properties and thus were not advanced further than animal testing. First, many of the discovered compounds are natural products, such as the rocaglate and silvestrol. Thus, their complex structures with multiple chiral centers make them particularly synthetically intractable and present a challenge for the intellectual property. ${ }^{9}$ Second, cell and animal models show the development of resistance quickly as silvestrol has a high affinity for the multidrug resistance efflux pump ABCB1 (p-glycoprotein,
MDR1). ${ }^{10}$ Thus, the discovery of novel scaffolds that inhibit eIF4A1 with unique mechanisms would facilitate bringing pharmacological inhibition of eIF4A1 into clinical practice.

eIF4A1 is known to undergo several conformational changes upon both nucleotide and RNA binding. Here we present multiple computational models of eIF4A1. Through molecular dynamics (MD) simulations, we are able to recreate the opening and closing of this DEAD-box helicase upon ATP binding. It has been reported that rocaglamide-A binds to the bimolecular cavity formed by eIF4A1 and RNA. ${ }^{11}$ However, detailed molecular modeling of the rocaglate binding sites as well as the molecular mechanism of action of other inhibitors such as elatol remains unknown, which hinders further optimization and development of these molecules. Furthermore, computational modeling of eIF4A1 with its natural ligands, ATP and RNA, has been limited by the absence of high-resolution crystal structures. Through the creation of several protein structure homology models of eIF4A1, we have been able to analyze important intra- and intermolecular interactions that influence enzyme activity. Subsequently, a

Received: May 28, 2021

Accepted: August 19, 2021

Published: September 15, 2021 
concerted effort of both large-scale virtual screening, machine learning, and extended molecular dynamics simulations was carried out to prioritize diverse small-molecule scaffolds, which may bind to and lock eIF4A1 in a closed conformation by competing with ATP in the nucleotide-binding site. We herein describe the pipeline of virtual screening and molecular modeling with experimental validation in biochemical and cell-based assays. We report the identification of several unique scaffolds that mimic 3D shape and electrostatics of ATP-type compounds and which, if optimized, may have potent antieIF4A1 and thus antitumor activity.

eIF4A1 is the prototypical DEAD-box helicase, aptly named for the conserved sequence D (Asp)-E (Glu)-A (Ala)-D (Asp), which is necessary for nucleotide binding at the active site. ${ }^{12-14}$ eIF4A1 catalyzes the ATP-dependent unwinding of 5' UTR on mRNA in preparation for ribosome binding and functions as the rate-limiting enzymatic step of cap-dependent protein translation, in particular for mRNAs with increased $5^{\prime}$ UTR length and complexity. The overall protein structure of a DEAD-box helicase consists of a helicase ATPase core formed by two RecA-like domains separated by a long, flexible linker. ${ }^{14,15}$ DEAD-box helicases are further characterized by the presence of several conserved motifs, which line the cleft between the two RecA-like domains and facilitate ATP binding, RNA binding, ATP hydrolysis, and RNA unwinding.

The complex functions of eIF4A are reflected in the structure of the $46 \mathrm{kDa}$ protein. ${ }^{11,12}$ eIF4A1 adopts several conformations, interacts with multiple proteins, and binds different biomolecules. The conserved motifs, found across all DEAD-box helicases, facilitate many of these functions, while the flexible linker gives the protein conformational plasticity. ${ }^{14}$ On the amino terminal domain, motifs I (AQSGTGKT) and II, also known as Walker A and B motifs (common to ATPases), coordinate the binding of ribose of ATP. The Qmotif (GFEKPSAIQQ), upstream of I and II, additionally possesses a conserved glutamine residue, which interacts with the adenine amino group of ATP. Motifs III, IV, and V, located on the C-terminal domain, are all involved in RNA binding. Motif VI (HRIGRGGR) contains three arginine residues, which extend into the interdomain cleft to coordinate the terminal phosphate of ATP. ${ }^{12,13}$

Structural studies of eIF4A1 have elucidated the molecular basis for the various conformations the protein adopts in the presence or absence of either RNA or nucleotide. Without any ligands, eIF4A1 adopts a variety of "open" conformations in which the two RecA-like domains are separated by the flexible linker and form few interdomain contacts. Therefore, open conformations represent an inactive state. Binding of ATP in the interdomain cleft, with the adenine interacting at the $\mathrm{N}$ terminal domain and phosphate groups extending to the Cterminal domain, results in a large conformational change to form a more compact, active structure. In this low-energy conformation, an intricate network of interdomain interactions is formed. RNA binding further stabilizes the closed conformation as RNA, like ATP, binding across both domains of the helicase. This bent geometry conformation is incompatible with the RNA duplex structure and thus the structure is "melted". A single-stranded RNA is released from the structure followed by ATP hydrolysis. Upon release of a phosphate group, the reopening of the interdomain cleft triggers the release of the second RNA strand, thus priming eIF4A1 for another cycle of ATP-dependent RNA unwinding. ${ }^{12-14}$ These effects occur within the context of the overall
eIF4F complex, including also the $5^{\prime}$ mRNA cap binder eIF4E and the scaffolding protein eIF4G (and variably the eIF4A1 cofactors eIF4B and eIF4H). Multiple copies of the eIF4A1 protein take part, jumping in and out of the RNA-protein complex with each cycle of ATP hydrolysis and resulting in RNA unwinding. ${ }^{16}$

Due to the various conformations that eIF4A1 is known to adopt, a virtual screening campaign, sampling this large conformational space, offers a potential strategy to identify potent inhibitors capable of binding the protein in its various states. A full high-resolution crystal structure of human eIF4A1 has recently been resolved with AMPPNP, RNA, and rocaglamide-A in the active, closed state (PDB: 5ZC9). ${ }^{11}$ However, no human structure exists with ATP-only or with no ligands in the open, inactive state. To evaluate ligand binding in the different conformations of eIF4A1, several homology models were built followed by docking and MD simulations.

\section{RESULTS}

elF4A1 Structural Models. PDB: $1 F U U$ is a crystal structure of the full-length yeast eIF4A1 protein. Sequence homology between the yeast and human protein is $63.5 \%$ (pBLAST). ${ }^{17}$ This structure is not cocrystallized with a nucleotide and thus is in the open, inactive conformation. In this conformation, the $\mathrm{N}$ and C-terminal domains are separated by a flexible linker (Pro231 to Arg247), adopting a structure resembling a barbell. A homology model to study the human form of eIF4A1, with 1FUU as a structural template, was created using prime ${ }^{18}$ (LLC) homology modeling wizard (A). The amino acid sequence for human eIF4A1 was obtained from Uniprot ${ }^{19}$ (ID P60842). Model refinement through ab initio loop modeling and energy minimization was performed, and Ramachandran plots confirmed energetically favorable and acceptable conformations. Extended molecular dynamic simulations were then run for $500-1000$ ns to assess local dynamics and overall protein stability (Supporting Information Figure 1). As expected, the open state of eIF4A1 undergoes many large fluctuations in $\mathrm{N}$ and $\mathrm{C}$ domains in the absence of ATP and RNA, with an average RMSD of $9 \AA$ (Supporting Information Figure 1). To assess the distance between $\mathrm{N}$ and C-terminal domains, the distance between the starting and ending residues of the flexible linker was measured throughout the MD simulation. The interdomain distance in the initial frame obtained from the homology model was about $38 \AA$ and ranges from 40 to $35 \AA$ throughout the MD simulation (Figure $2)$. The distances between motifs $\mathrm{Q}$ and I ( $\mathrm{N}$ terminus) with motifs V and VI ( $\mathrm{C}$ terminus) remain large; without nucleotide or ligand binding, these residues do not come into close contact with one another. Consistent with experimental data, this model confirms that no interdomain contacts are observed in the absence of nucleotide or RNA. The same analyses and molecular dynamics simulations were also run with the original crystal structure 1FUU to compare the behavior with our homology model (Supporting Information Figure 2). As expected and similar to our model, the original yeast structure (without ATP) remains in the open, inactive conformation with large interdomain distances.

Docking grids were then created using the centroid of coordinates of residues known to be involved with nucleotide binding, such as those in the Q-Motif and Motifs I and II. ATP was then docked in 10 different grids with restrictions made so that only poses containing a hydrogen bond with the amino group (N6 or N7) of ATP adenine were considered. The 

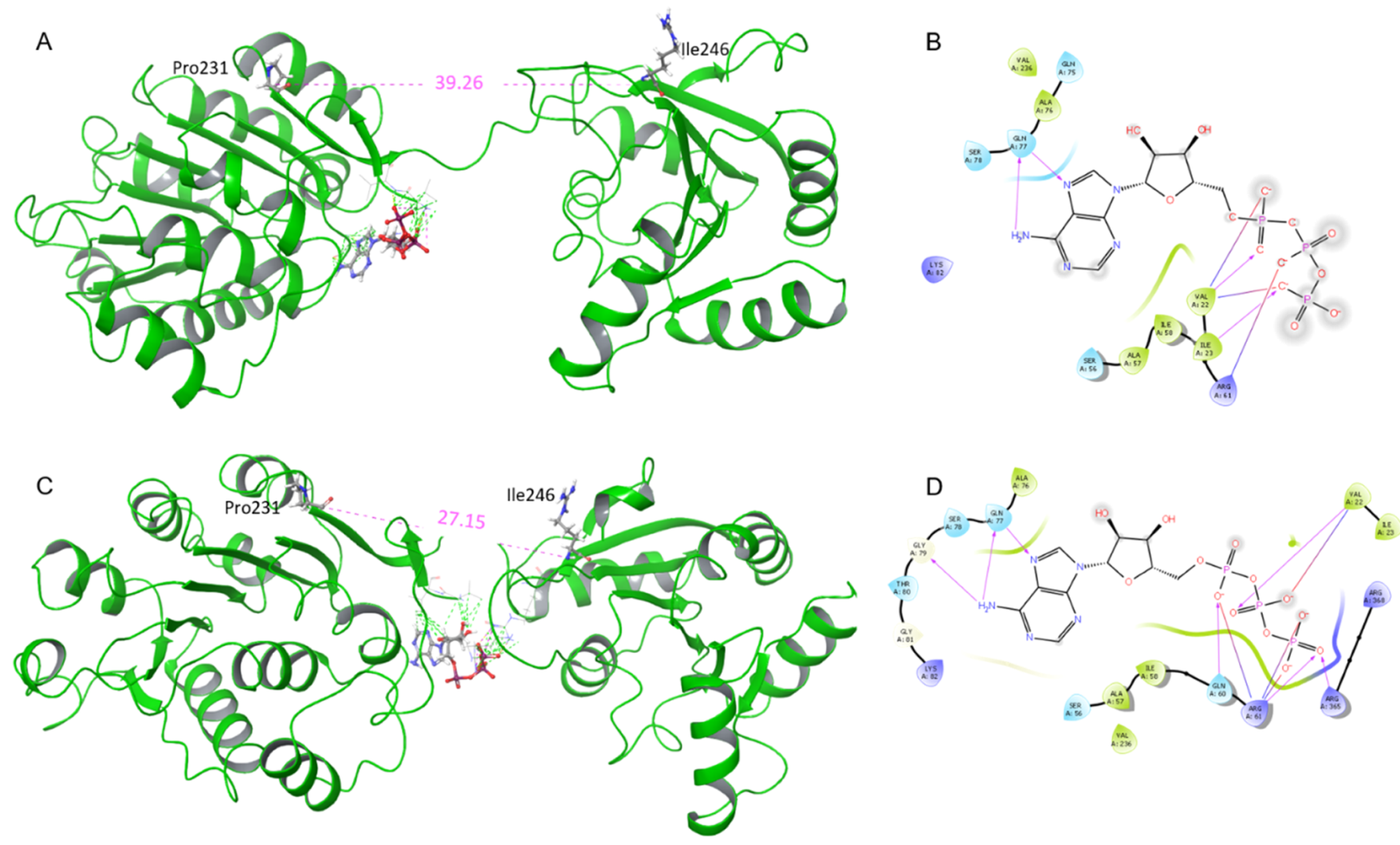

Figure 1. 1FUU eIF4A1 homology model. (A) ATP is docked in the open state of the 1FUU homology model. ATP initially binds in the Nterminal domain with contacts between the adenine moiety and motifs $Q$ and I. The interdomain distance (Pro231 to Ile246) in the open conformation is measured to be 39.26 angstroms. (B) 2D representation of ATP docked in the N-terminal domain of the open-conformation eiF4A1 model with hydrogen bonds to a conserved glutamine residue and several residues in the interdomain linker. (C) After an extended molecular dynamic simulation, the 1FUU homology model adopts a closed conformation, with the interdomain distance between Pro231 and Asp247 reduced to $27.15 \AA$ A. (D) ATP retains hydrogen bonds to the conserved glutamine residue in the N-terminal domain; however, terminal phosphates of the ATP extend to the C-terminal domain with key interactions occurring between arginine residues in motif IV.

highest scoring poses with all required binding contacts were then selected for further analysis via the MD simulation. Analysis across all poses confirmed a consensus that the adenine base fits into a pocket built of residues conserved in all DEAD-box proteins. The adenine nitrogens interact with conserved glutamine residues in the I and Q motifs, while $\pi-\pi$ stacking is observed in some poses with conserved phenylalanine residues and various interactions of the terminal phosphate groups occur with residues in the linker domain (Val22, Ile23) (Figure 1B). Top poses were selected for the MD simulation, which would optimize binding interactions of ATP and eIF4A1 through stochastic space sampling of poses (see Methods).

MD simulations were initially used to confirm correct binding poses as the ATP remains stably in the binding site throughout the simulation (see Methods). In the ATP-bound state, R365 and R362 from conserved motif VI (HRIGRGGR) formed salt bridges with the g-phosphate of ATP, and the residues involved in nucleotide binding remained stable during the entire simulation. The system quickly equilibrates to ATP binding and adopts a lower energy closed conformation, with the ATP phosphate groups extending to the $\mathrm{C}$-terminal $\mathrm{V}$ and VI motifs, bringing the $\mathrm{C}$ - and $\mathrm{N}$-terminal domains in close proximity to one another (Figure 1C, D). The linker distance decreases from an average of 39 to $27 \AA$ and the system converges at a lower energy, closed conformation (Figure 1C,1D). Additionally, several sustained interdomain hydro- phobic interactions exist in the closed, active state, between loops of VI motifs and C-terminal motifs. In the closed model, the linker also appears to fold in on itself, with multiple intradomain interactions. To test the stability of this state of the protein, a longer MD simulation was run for $3 \mathrm{~ms}$ during which the ATP remains bound to the protein in the closed conformation, with key interactions sustained and an average linker distance reduction of $11 \AA$ (Figure 2). RMSF analysis of individual residues, which had contact with ATP in the ATPdocked model (Motifs I, Q, VI), compared to those same residues in the model without ATP, reveals significant differences (Supporting Information Figure 1). This is to be expected as nucleotide binding elicits large fluctuations in the C- $\alpha$ backbone on key residues in the protein. To confirm this interaction, the MD study was repeated in the original yeast model where similar domain closure occurred (Supporting Information Figure 2). Prime MMGBSA binding free energetic analysis of the 1FUU system in the open versus the closed position with ATP bound demonstrates a decrease in total free energy (Table 1), with an increase in favorable hydrophobic and Coulombic interactions upon ATP binding.

Previous attempts to model eIF4A1 conformational changes focused only on the yeast protein, not a homology model of the human protein. Furthermore, the study only reports MD simulations of $2 \mathrm{~ns}^{20}$ Thus, this is the first large-scale extended molecular dynamics study of a model of the openconformation human eIF4A1 protein. At the start of this 
1FUU: elF4A-I Homology MD

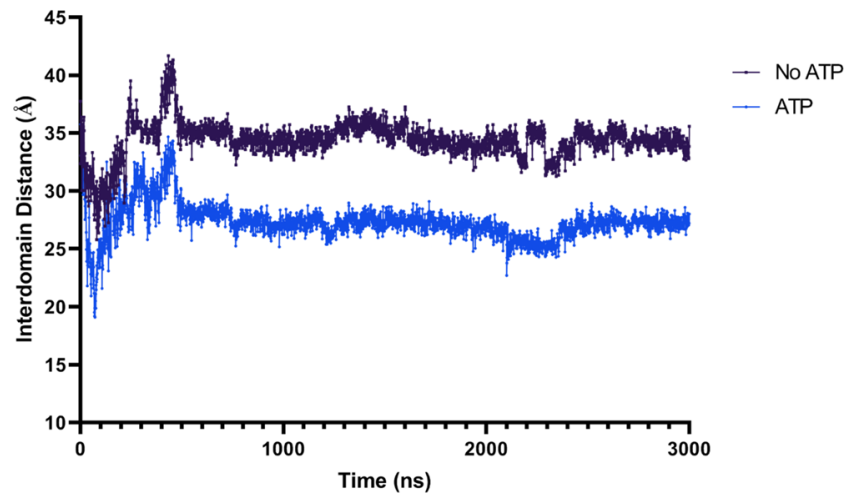

Figure 2. Interdomain distance during MD simulations of the 1FUU model. Extended MD simulations of the 1FUU homology model were run for $3 \mathrm{~ms}$ in both the presence and absence of ATP. With ATP bound in the $\mathrm{N}$-terminal domain, interdomain closure is quickly observed within the first $10 \mathrm{~ns}$. The system equilibrates to an energy minimum at $60 \mathrm{~ns}$ with domain closure and is sustained for the entirety of the $3 \mathrm{~ms}$ simulation, the system converging at an interdomain distance of around $27 \AA$. Without ATP, the system remains open with an interdomain distance around $35 \AA$.

Table 1. Energy Analysis for the 1FUU Homology Model ${ }^{a}$

\begin{tabular}{cccccc}
$\begin{array}{c}\text { homology } \\
\text { model }\end{array}$ & ligand & pose & $\begin{array}{c}\text { prime } \\
\text { Coulomb } \\
(\mathbf{k J} / \mathbf{m o l})\end{array}$ & $\begin{array}{c}\text { prime vdW } \\
(\mathbf{k J} / \mathbf{m o l})\end{array}$ & $\begin{array}{c}\text { prime } \\
\text { energy } \\
(\mathrm{kJ} / \mathbf{m o l})\end{array}$ \\
\hline 1FUU & ATP & open & -12081 & -1038 & -12010 \\
1 FUU & ATP & closed & -12519 & -1140 & -12581 \\
\hline
\end{tabular}

${ }^{a}$ ATP was docked into the 1FUU model and molecular dynamics were run so that the structure would adopt a closed conformation. Prime was used to calculate the total system energy, VdW energy, and Coulombic energy in the initial structure (open) and again at the end of the MD simulation in the closed structure. ATP binding promotes domain closure and interdomain interactions causing a decrease in the overall system energy.

project, a structure of human eIF4A1 in a closed, active conformation had not been resolved. A protein BLAST query using the human eIF4A1 sequence revealed that homologous human DEAD-box protein, eIF4A3 (66\% homology), had been crystallized with the nucleotide (ANP; PDB: 2JOS). ${ }^{21}$ Therefore, a homology model was generated using this as a structural template. First, MD simulations were run on the native structure to evaluate nucleotide-binding stability and domain flexibility. To assess the structure's ability to undergo conformational changes, ANP was removed from the binding site and MD simulations were run for 500-1000 ns. As predicted, the structure transformed from a "closed" active state to an open inactive state, measured by increases in the distance between the two RecA-like domains via residues of the flexible linker as done with the 1FUU model (Supporting Information Figure 3). ATP was then redocked into the final frame of the MD simulation of 2J0S (now in the open state) and domain closure was observed. These same experiments were conducted in the eIF4A1 2J0S homology model with similar results although with less significant changes of the domain conformation (Figure 3). Binding free energy calculation using Prime MMGBSA confirmed that the 2J0S homology model assumes a lower-energy state upon ATP binding and domain closure facilitated by numerous interdomain and ligand-domain contacts (Table 2). Our
2J0S: elF4A-I Homology MD

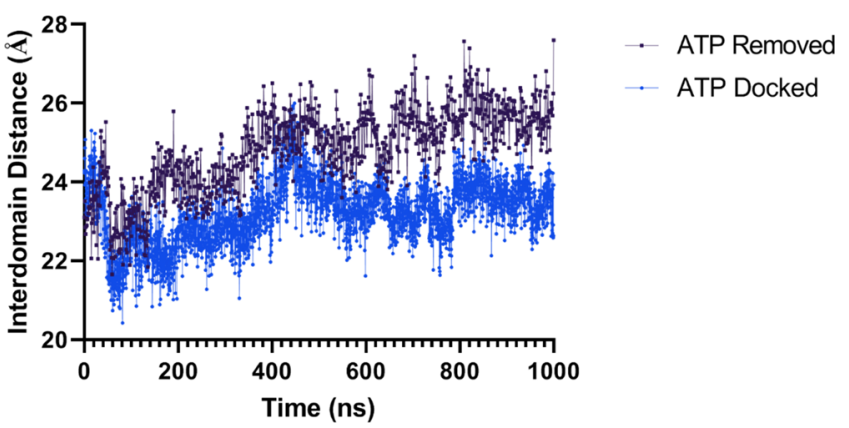

Figure 3. 2JOS homology model analysis. ATP was removed from the active site of the 2JOS homology model and a molecular dynamics simulation was run for $1 \mathrm{~ms}$. The protein quickly adopted an open conformation with an increase in interdomain distance from 22 to 27 Å. Docking ATP back into the model in the final frame and running the simulation for an additional $1 \mathrm{~ms}$, the system closes again and converges at an energy minimum with an average interdomain distance of $23 \AA$.

Table 2. Energy Analysis for the 2J0S Model ${ }^{a}$

\begin{tabular}{ccccc}
$\begin{array}{c}\text { homology } \\
\text { model }\end{array}$ & ligand & $\begin{array}{c}\text { delta energy } \\
(\mathbf{k J} / \mathbf{m o l})\end{array}$ & $\begin{array}{c}\text { delta vdW } \\
(\mathbf{k J} / \mathbf{m o l})\end{array}$ & $\begin{array}{c}\text { delta Coulomb } \\
(\mathbf{k J} / \mathbf{m o l})\end{array}$ \\
\hline $2 \mathrm{j} 0 \mathrm{~s}$ & $\begin{array}{c}\text { ATP } \\
\text { removed }\end{array}$ & 134.44 & 18.23 & 99.84 \\
$2 \mathrm{j} 0 \mathrm{~s}$ & $\begin{array}{c}\text { ATP } \\
\text { redocked }\end{array}$ & -585.07 & -367.68 & -220.59 \\
& & & &
\end{tabular}

${ }^{a}$ ATP was removed from the 2 JOS homology model and molecular dynamics were run so that the structure would adopt an open conformation. Prime MMGBSA was used to calculate the change in total energy, vDw energy, and Coulombic energy from the initial structure. ATP was then redocked into the model and MD simulations were run to promote domain closure. Prime was then used to calculate the change in energies upon domain closure. This demonstrates the creation of a dynamic eIF4A1 model and that ATP binding causes major energetic transformations to a low energy, stable system.

model thus reproduced the dynamics of domain closure upon ATP binding and opening upon release. The model therefore appeared suitable to evaluate if compounds docked in one of the "closed" conformations could keep the structure closed and compact. Our theory was that weaker binders would be more likely to leave the binding site during a $\mathrm{MD}$ simulation accompanied by unraveling of the structure into an open conformation.

A third model, from PDB: 5ZC9, was the first full-length human eIF4A1 structure crystallized with RNA, AMPPNP, and the rocaglate prototype inhibitor rocaglamide-A. ${ }^{11}$ The 5ZC9 crystal structure was used to confirm the proper binding pose of ATP in eIF4A1, which we had previously attempted to model in the two dynamic homology models (1FUU and 2J0S). This model was also used for binding pose optimization and molecular dynamics analysis of the top hits identified in the biochemical and cell-based screens.

In summary, we have developed and analyzed three distinct models of eIF4A1, which are able to accurately portray the conformational and energetic changes associated with nucleotide binding. Leveraging these data, we set forth on a virtual screen to identify inhibitors of eIF4A1 at the nucleotide-binding site. We hypothesized that compounds predicted to bind to eIF4A1 in this site reduce system energy 


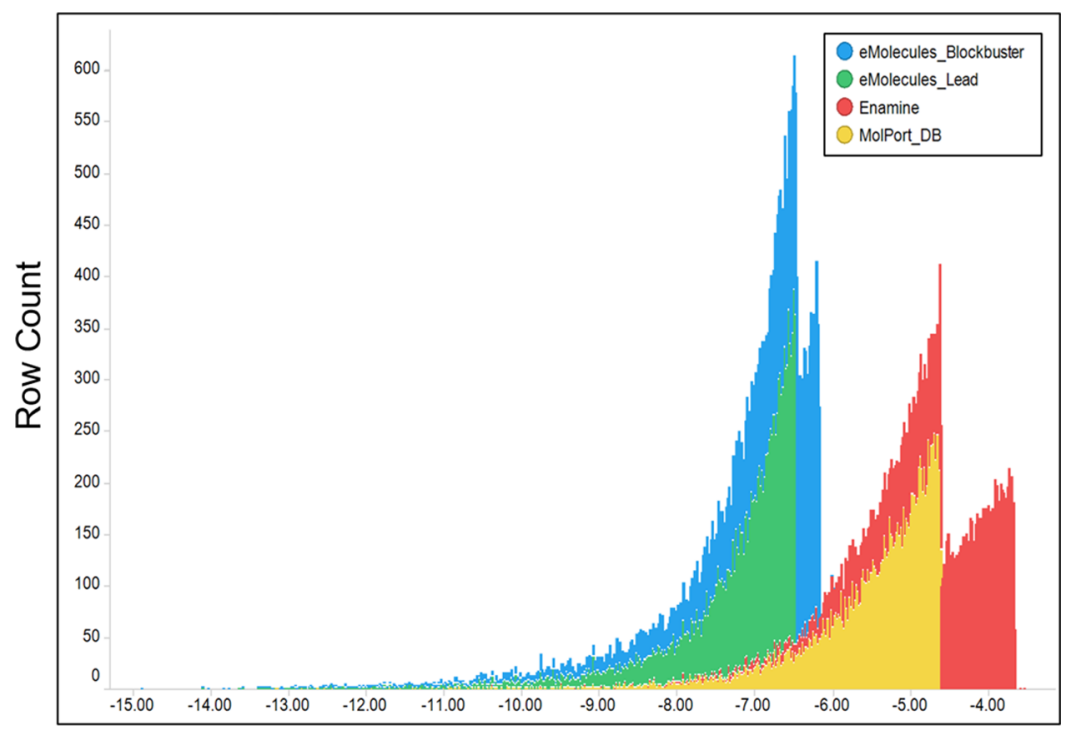

HYBRID ChemGauss4 Docking Score

Figure 4. Distribution of HYBRID scores by the small-molecule database. Top $25 \%$ of scores were selected for output so there is a sharp right-sided decline for each curve. Two visible peaks in blue and red represent 2J0S and 1FUU, with HYBRID from the 2J0S models trending toward better, more negative docking scores. For reference, ATP scores on average -7 in the 1FUU model, while it scores on average -9 in the 2 J0S model. On average, compounds filtered from the eMolecules Blockbuster library scored better, likely because they are larger and more drug-like versus smaller lead-like compounds in the other libraries.

and lock eIF4A1 in a closed conformation during MD analyses and may prevent cycling through ATPase and RNA unwinding activities in biochemical and in vitro assays.

Virtual Screening and MD Simulations to Identify elF4A1 Inhibitors: Nucleotide-Binding Site. Leveraging the results data from our MD simulations analyses above, we sought to perform a virtual screening campaign where we prioritized compounds that (1) bound to eIF4A1 in the nucleotide-binding site (in both open and closed-conformation models) and (2) could stabilize the protein in a closed, lowenergy state. We hypothesized that potent inhibitors would out-compete ATP in the binding site and would thus prevent the cycling of ATPase and helicase activity.

Trajectories from each model's MD simulation with ATP bound were clustered by frames based on RMSD. Ten representative ligand-complex poses were exported from the 1FUU and 2J0S model trajectories. Each unique pose of ATP was then exported from the complex and used to screen several virtual libraries to identify small-molecule compounds with both similar three-dimensional shape and electrostatics. Compounds from the original 2D databases were prepared by first generating all tautomers and ionization states from using QUACPAC (OpenEye) followed by generating 100$1503 \mathrm{D}$ conformers using OMEGA. ${ }^{22}$ Using fastROCS ${ }^{23}$ with GPU technology, over 20 million compounds from Enamine, MolPort, and eMolecules commercially available libraries were screened and prioritized by Tanimoto ${ }^{24}$ color score, Tanimoto shape score, and Tanimoto combo score-an aggregate of color and shape scores. In addition, a small library of ATPcompetitive kinase inhibitors was also screened from SelleckChem. Subsets of compounds from each database were created for docking using physiochemical properties (see Methods). The top $10 \%$ compounds based on Tanimoto combo scores from each database were then used to dock across all 10 grids of each eIF4A1 structural model using HYBRID docking. ${ }^{25}$
Docking scores were first analyzed separately per model (open: 1FUU and closed: 2J0S), with all scores from each of the 10 grids being aggregated and averaged (Figure 4). Compounds were clustered by topological similarity and clusters with the highest average HYBRID docking scores were chosen for further analysis. Using Maestro, which allows for interactive $3 \mathrm{D}$ analysis of binding poses, the top 5000 scoring compounds from the top clusters from HYBRID were docked again across all models. Compounds were again rank ordered by individual docking scores and cluster average scores. Top compounds of the best clusters were manually analyzed for key hydrogen bonding interactions. In the closedconformation model (2J0S), compounds with hydrogen bonds in the $\mathrm{Q}, \mathrm{V}$, and VI motifs, particularly to conserved glutamine residues in the $\mathrm{N}$-terminal domain and arginines in the $\mathrm{C}$ terminal domain, were prioritized. In the open-conformation model (1FUU), flexible compounds with a strong hydrogen bond in the $\mathrm{Q}$ I, or Ia motifs on the N-terminal were prioritized. Some larger compounds, for example, could span the distance between domains and interact with key arginines in the $\mathrm{V}$ and VI motifs in the 1FUU docking models. The distribution of docking scores was different for each model and each database, with compounds scoring better in the closed model due to increased ligand-protein interactions. However, it was reasoned that compounds with higher average scores between each model were the best candidates to move forward with biochemical screening. One hundred unique compounds with differing scaffolds were chosen for the initial in vitro experiments.

Biochemical and Cell-Based Screens for elF4A1 Inhibitors. Sixty-one compounds (named UM98-UM158) were identified from virtual screening campaigns targeting the nucleotide-binding site of eIF4A1 and were purchased for in vitro screening (Supporting Information Table 1). Compounds were tested in both a malachite-green based, ATPase biochemical assay and in a cell-based viability assay (see 
<smiles>CC(NC(N)=O)C(=O)N1CCN(c2ccc(C#N)cc2F)CC1</smiles>

C

B<smiles>O=C([O-])CCC(=O)N1CCN(c2ccc(Cl)c(Cl)c2)CC1</smiles>

Figure 5. 2D representation of phenyl-piperazine hit compounds: (A) UM127, (B) UM139, (C) UM162, and (D) UM167.
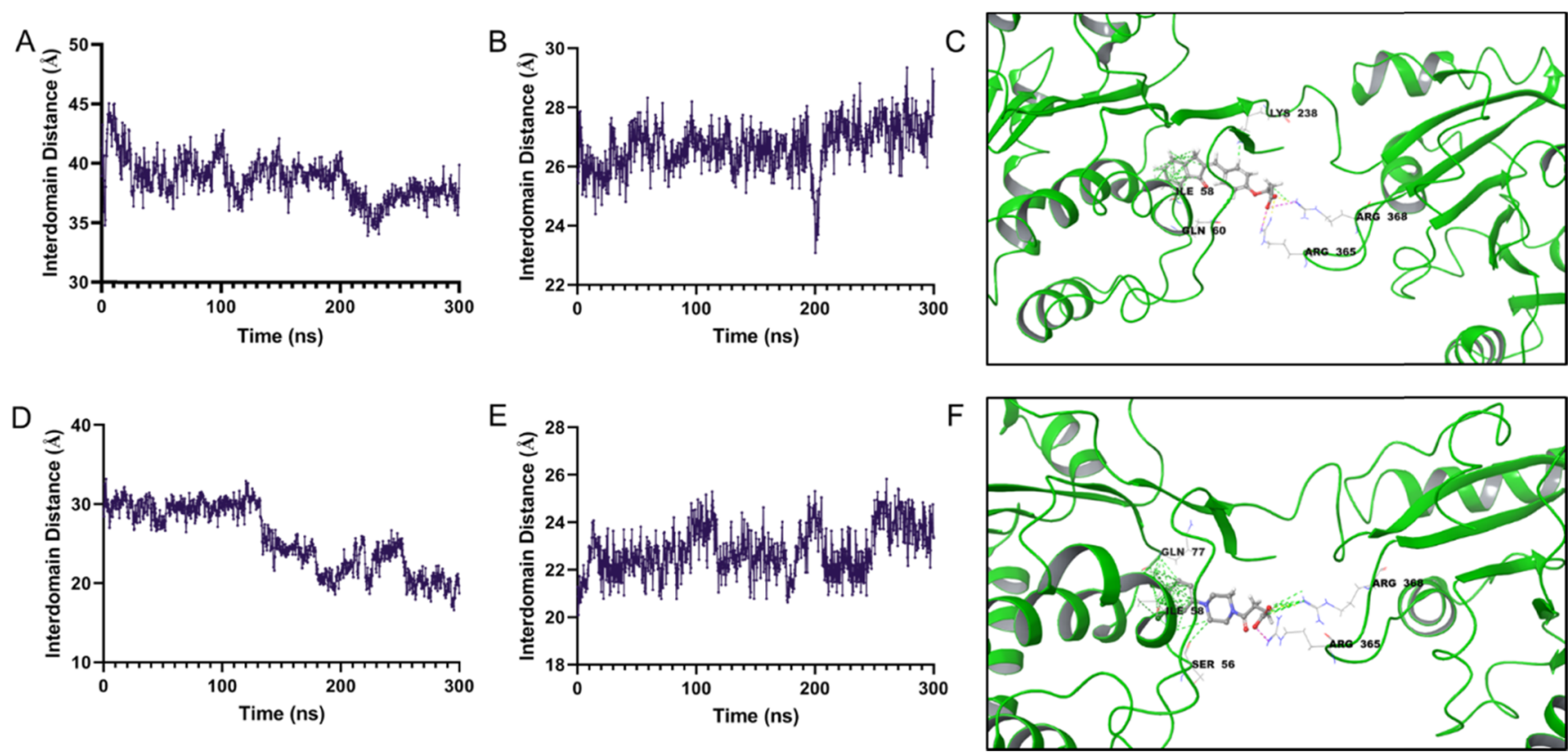

Figure 6. Molecular dynamics analysis of top hits. Interdomain distance analysis of UM127 in the 1FUU (A) and 2J0S (B). UM127 causes large conformational changes in both models, which leads to partial domain closure. (C) The model predicts that UM127 promotes intralinker contacts. UM139 in the 1FUU (D) and 2J0S (E) leads to full domain closure in the 1FUU model and maintains domain closure in the 2J0S model. (F) Binding post of UM139 shows similar contacts seen with ATP including bonding with Arg365 and Arg368.

Methods, Supporting Information Table 1, Figure 4). For the biochemical screen, compounds were tested initially at high concentrations of $250 \mathrm{mM}$ and then tested in a 12-point doseresponse using 2 -fold serial dilutions. Thirteen of these compounds were considered weak hits (IC50 >125 mM, $<250 \mathrm{mM}$ ), showing some inhibitory effect at concentrations of $125 \mathrm{mM}$, while the majority of the compounds had little to no inhibitory activity at concentrations less than $250 \mathrm{mM}$. Twelve of the 13 weak hit compounds consisted of the same 1phenyl-piperazine core scaffold present in compounds UM127 and UM139 (Figure 5). Quercetin (UM107) was also identified as a weak hit from our screen, which also included a subset of compounds known to be ATP-competitive kinase inhibitors. This polyphenol is a known anticancer agent, with multiple in vitro targets such as PI3K and eIF2. Doseresponse data show an $\mathrm{IC}_{50}$ of eIF4A1 $\sim 200 \mathrm{mM}$, while cellbased assays give an $\mathrm{IC}_{50}$ of $25-50 \mathrm{mM}$. However, due to the promiscuous nature of such polyphenol scaffolds, further analysis was not pursued. ${ }^{26,27}$

MD simulations of $300 \mathrm{~ns}$ were used to evaluate the binding and energetics of both the phenyl-piperazine scaffold hits in addition to compounds that showed no activity in the initial screens. Compound UM139 demonstrated favorable molecular dynamics, with interdomain closure noted in 1FUU and 2J0S models (Figure 6). UM127 scored well in docking models, was a weak hit $\left(\mathrm{IC}_{50} 125 \mathrm{mM}\right)$, and had some cytotoxic effect but displayed only transient domain closure (Figure 6). Most of the compounds with no activity in the biochemical screen did 
Table 3. Comparing Physiochemical Properties of Original and Phenyl-Piperazine Compounds ${ }^{a}$

\begin{tabular}{|c|c|c|c|c|}
\hline property & average original & range original & average analogues & range analogues \\
\hline molecular weight $(\mathrm{g} / \mathrm{mol})$ & 323.89 & $244.24-461.53$ & 334.28 & $276.33-392.36$ \\
\hline hydrogen-bond donors & 1.81 & $0-4$ & 1.59 & $1-3$ \\
\hline hydrogen-bond acceptors & 6.34 & $3-10$ & 8.09 & $6-11$ \\
\hline $\mathrm{Q} \log \mathrm{Po} / \mathrm{Pw}$ & 1.74 & $-0.303-3.898$ & 1.11 & $-0.63-3.52$ \\
\hline QPPCaco (not cell-permeable $<25$, very permeable $>250$ ) & 83.64 & $2.18-619.02$ & 239.13 & $4.20-1430.78$ \\
\hline SASA & 580.44 & $464.52-721.04$ & 601.90 & $549.92-672.68$ \\
\hline PSA & 109.40 & $74.39-164.79$ & 113.02 & $67.52-153.26$ \\
\hline
\end{tabular}

${ }^{a}$ Properties calculated using QikProp (Schrödinger, LLC, New York, NY, 2020).
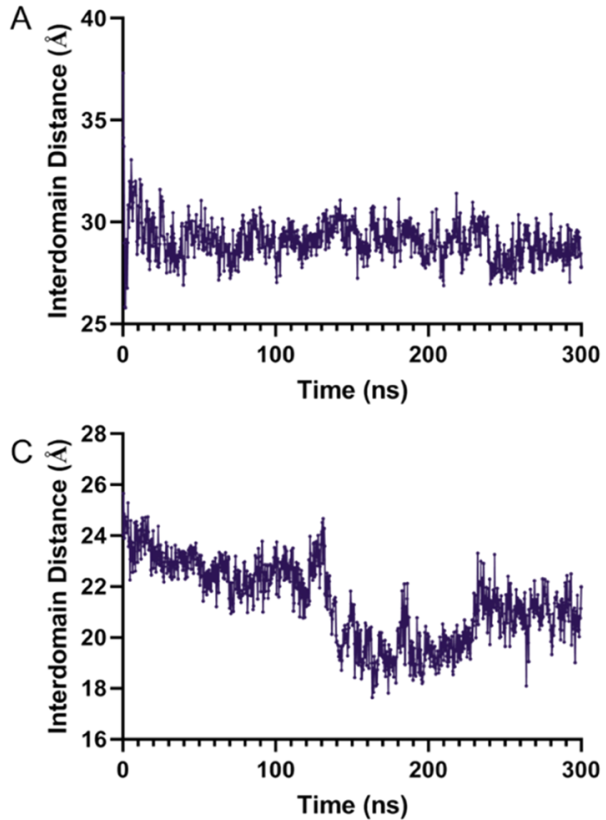

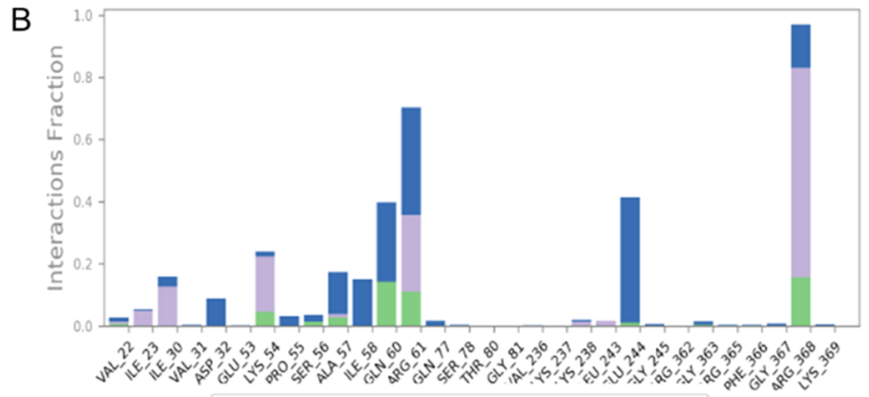

H-bonds $\mathrm{H}$ Hydrophobic $\square$ lonic $\square$ Water bridges

D

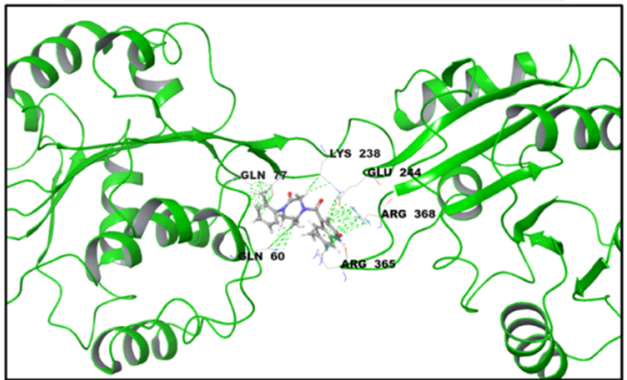

Figure 7. Molecular dynamics analysis of UM167. UM167 was the most potent hit identified in the biochemical screen. MD simulation in the 1FUU model (A) shows rapid domain closure with sustained interactions with Arg368, conserved glutamine Gln60, and a glutamic acid residue Glu244 in the interdomain linker (B). (C) UM167 also maintains domain closure in the 2J0S model. (D) Binding poses reveal that the UM167 binding leads to domain closure with multiple interdomain and protein-ligand contacts.

not have stable interactions during molecular dynamic simulations and subsequently fell out of the binding pockets in both models. To further explore the possibility of the phenyl-piperazine scaffold present in many of the weak hits, another fastROCS ${ }^{23}$ shape screen of three virtual databases (Enamine, eMolecules, and MolportDB) was performed using UM139 and UM127 (in their conformations after MD simulation) as reference structures (Figure 5). After docking in both models and ranking as described previously, 11 additional compounds (UM159-UM169) were prioritized and purchased for evaluation. All compounds contained the phenyl-piperazine scaffold and (compared to the original reference compounds) were of slightly higher molecular weight, more hydrophilic and cell-permeable, and contained more HBDs and HBAs, which resulted in better docking scores compared to UM127 and UM139 (Table 3, Supporting Information Table 1). MD simulations in 1FUU and 2J0s also demonstrated fast domain closure and stable binding in the nucleotide site for compounds UM161, UM162, and UM167 (Figure 7), with notable interactions similar to that of ATP in the 1FUU model, including contacts with Lys54, Gln60, Glu244, and Arg368. An improvement in inhibition was also noted in the biochemical screen, with $\mathrm{IC}_{50}$ values for the enriched phenyl-piperazine analogue compounds ranging from $\sim 64$ to $125 \mathrm{mM}$; compound UM159 was excluded from analysis due to solubility issues (Table 4). UM167 was the

Table 4. Biochemical Activity Data for Phenyl-Piperazine Analogue Compounds

\begin{tabular}{cc} 
compound ID & biochemical IC50 $(\mu \mathrm{M})$ \\
UM-160 & $110.4 \pm 11.62$ \\
UM161 & $107.3 \pm 12.24$ \\
UM162 & $82.59 \pm 6.19$ \\
UM-163 & $124.5 \pm 15.18$ \\
UM-164 & $110.4 \pm 9.30$ \\
UM-165 & $>125$ \\
UM-166 & $>125$ \\
UM167 & $69.51 \pm 5.34$ \\
UM-168 & $113.6 \pm 11.88$ \\
UM169 & $>125$ \\
\hline
\end{tabular}

most potent inhibitor identified from the nucleotide-binding site campaign with an $\mathrm{IC}_{50}$ of $69.51 \pm 5.34 \mathrm{mM}$ followed by UM162 with an $\mathrm{IC}_{50}$ of $82.59 \pm 6.19 \mathrm{mM}$ (Figure 5). Prime MMBSA calculations of compounds from the phenylpiperazine scaffold screen demonstrated that the more potent compounds (UM167, UM162) have lower binding free 
energies than the initial weak hits (UM127, UM139) (Table 5).

Table 5. Changes in System Energy Following Compound Docking and Molecular Dynamics ${ }^{a}$

$\begin{array}{ccccc}\text { model } & \text { compound } & \begin{array}{c}\text { delta energy } \\ (\mathbf{k J} / \mathbf{m o l})\end{array} & \begin{array}{c}\text { delta VdM } \\ (\mathbf{k J} / \mathbf{m o l})\end{array} & \begin{array}{c}\text { delta Coulomb } \\ (\mathbf{k J} / \mathbf{m o l})\end{array} \\ \text { 1FUU } & 167 & -183.96 & -75.15 & -44.69 \\ \text { 1FUU } & 162 & -173.49 & -88.03 & -61.9 \\ \text { 1FUU } & 157 & -113.39 & -20.4 & -68.4 \\ \text { 1FUU } & 139 & -5.52 & -41.22 & -21.91 \\ \text { 1FUU } & 127 & -71.86 & -16.29 & -26.7\end{array}$

${ }^{a}$ Prime MMGBSA was used to calculate the change in total energy, $\mathrm{vDw}$ energy, and Coulombic energy at the beginning (open conformation) and end of molecular dynamic simulations (closed conformation). In all top compounds identified in the virtual screen and biochemical screen, there is a marked reduction in overall energy in the system representative of favorable ligand-protein interactions and interdomain interactions reflected in the promotion of stabilization of the protein in the closed conformation. The most potent compound, UM167, demonstrates the greatest reduction of total energy upon binding the $1 \mathrm{FUU}$ model.

Additional docking and molecular dynamics analyses were carried out in the 5ZC9 human eIF4A1 model with RNA to develop binding hypotheses and optimization strategies for the most potent hits. UM167 is predicted to bind in the ATPbinding site with interactions with residues in key motifs in both the $\mathrm{N}$-terminal and $\mathrm{C}$-terminal domains. For example, there are $\pi$-stacking interactions between a benzyl group and Phe52 and strong interactions with the conserved glutamine residue (Figure 8). Similarly, there are interactions with Arg362 and Arg365 with the nitrogen in the heterocycle and many water bridges and hydrogen bonds with residues in motifs I and II. MD simulation for 300 ns showed sustained interactions at all residues identified in the docking model, suggesting that the compound remains stable in the binding site. UM162, another more potent hit, displayed different binding predictions during the MD simulation mostly dominated by ionic interactions and coordination with an active site magnesium (Supporting Information Figure 5).

Compounds from the nucleotide-binding site screen had an average molecular weight of $322 \mathrm{~g} / \mathrm{mol}$, possessing many lead- like qualities including low molecular mass, ClogP, and few hydrogen-bond donors and acceptors. However, the analysis from QikProp revealed many of these compounds suffered from poor cell permeability (QPPCaCo, Supporting Information Table 1). Therefore, it was no surprise that many of the compounds that were identified as hits in the biochemical screen had little to no activity in cell-based viability assays (Supporting Information Figure 4). Cell-based viability assays of UM98-UM158 revealed some cytotoxic effects from UM107, UM108, UM109, UM127, and UM157, with $\mathrm{IC}_{50}$ 's ranging from 25 to $50 \mathrm{mM}$. UM127 with the piperazine scaffold, though weakly potent in inhibiting eIF4A1 in biochemical screens, had some cytotoxic effects in the cellviability assays, perhaps representing a promising starting point which can be pursued further via medicinal chemical optimization.

\section{DISCUSSION}

Preclinical studies-biochemical, cellular, and animal models-over the past decade have confirmed the hypothesis of targeting protein translation initiation through eIF4A1 inhibition to elicit antitumor toxicity while maintaining a therapeutic window for normal cells. eFFECTOR Therapeutics recently began a first-in-human phase I clinical trial with the compound eFT226 (Zotatifin), ${ }^{28}$ a rocaglate-like compound that similarly clamps eIF4A1 onto polypurine-rich mRNA transcripts. Additionally, rocaglates have been shown preclinically to be effective against viral, parasitic, and fungal infections. Recently and most notably, rocaglates were shown to effectively inhibit replication and infectivity of SARS-COV2 , which is responsible for causing COVID-19. ${ }^{29}$ While we anxiously await results from this clinical trial, we understand there still remains an unmet need to generate more potential clinical candidates for this class for treatment of cancer and potentially for infectious diseases that hijack the host cell's translational apparatus.

Our novel approach takes advantage of three key fields of biomedical research to most effectively identify and test lead and lead-like compounds: computational biology, computational chemistry, and molecular biology. We used computational modeling to jumpstart the drug discovery process for eIF4A1 inhibitors. With limited crystal structures available, we generated homology models of the protein that accurately
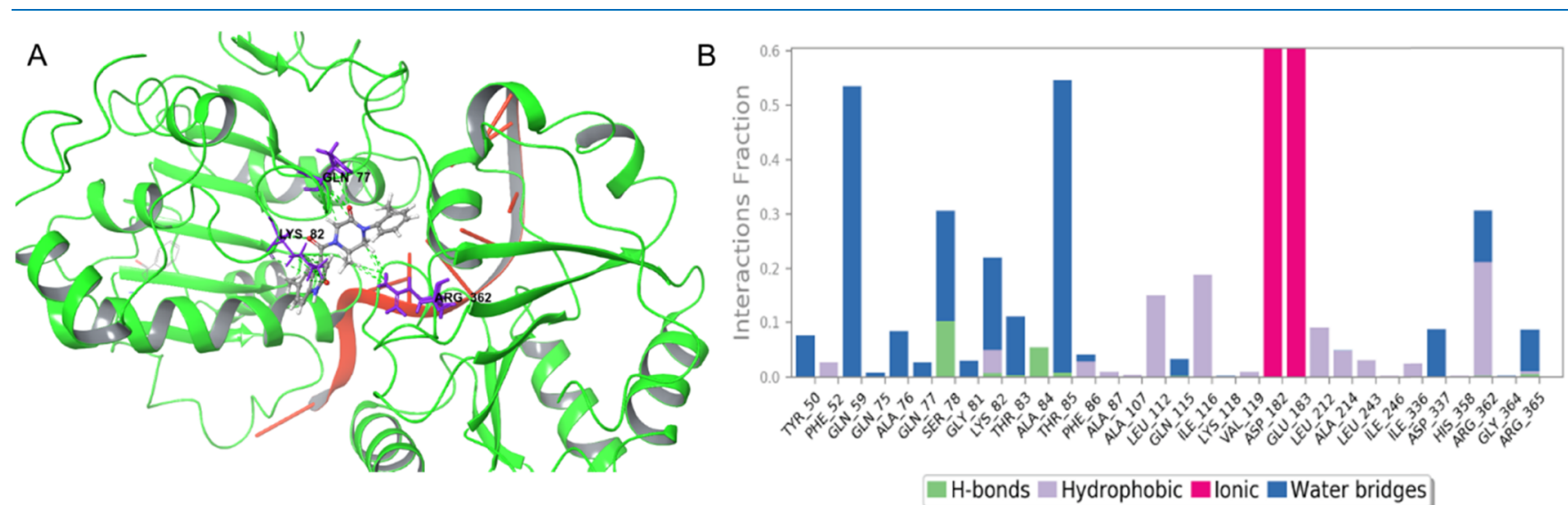

Figure 8. Binding hypothesis of UM167 in the human eIF4A1 model (5ZC9). UM167 binds in the interdomain cleft of eIF4A1 with key interactions with conserved glutamines Gln59 and Gln77, active site Lys81, and motif VI Arg362 (A, B). Molecular dynamic simulations in the 5ZC9 model were stable and UM167 locked the model in the closed conformation with RNA also remaining in the interdomain cleft. 
reproduce known activity in molecular dynamics simulations. This approach enabled us to employ an extensive virtual screen canvassing nearly 20 million commercially available compounds and docking into multiple poses, begetting a more physiological model. We hypothesized that inhibition of eIF4A1 by screening for inhibitors of the nucleotide (ATP)binding site may identify compounds that inhibit enzymatic activity through ATP competition and stabilization in a low energy, closed conformation. Prioritization of compounds and further virtual assessment through molecular dynamics simulations enabled higher confidence when selecting compounds for purchasing and biochemical/cellular testing. Our initial results suggest that molecular dynamics analyses using multiple dynamic models of eIF4A1 to simulate physiological domain closure and interaction may help to enrich and prioritize compounds for biochemical screening. We are limited, however, in the computational abilities to run multiple extended molecular dynamic simulations in parallel on multiple compounds across several structural models. Therefore, it is necessary to use other high throughput methods of enrichment, such as docking in parallel across multiple protein conformations with HYBRID, ${ }^{25}$ clustering of compounds by structure and docking score, filtering on the basis of predicted physiochemical properties with tools such as QikProp, and evaluating binding free energy changes upon binding and molecular dynamics using MMGBSA calculations. $^{30,31}$ For example, in our small pilot study, we observed a trend that compounds with higher docking scores that can reproduce domain closure and demonstrate a large reduction in free energy upon binding and stabilization in silico are more potent in biochemical screens. In future studies, we may prioritize compounds for in vitro testing with certain physiochemical properties such as molecular weights $>350$ $\mathrm{g} / \mathrm{mol}, \mathrm{HB}$ acceptors $>7$, and QPPCaCo $>25$. Additionally, a higher-order predictive machine learning model may be developed using the docking, shape screen, and MD simulation results along various other descriptors to accelerate and scale up the computational screen.

We believe we have established a successful method for identifying lead-like eIF4A1 inhibitors that can be further optimized using medicinal chemistry. The identified piperazine scaffold is chemically tractable and possesses drug-like properties and it is also found in reports of eIF4A3 inhibitors in addition to other RNA helicase inhibitors. UM127, UM139, UM162, and UM167 are all lead-like with molecular weights of less than $400 \mathrm{~g} / \mathrm{mol}$. Thus, they have the potential for medicinal chemistry optimization including increasing hydrophobicity, molecular weight, and other key properties to improve solubility, binding, potency, and cell permeability. Out results support for the application of computational modeling and, specifically for this target, combining homology modeling, docking, molecular dynamics simulations, and large-scale shape-based screening to guide hit identification. Refinement of our model and further exploration of the available space of small molecules and their binding modalities will likely generate additional leads and new structure-activity relationship insights.

\section{METHODS}

Protein Model Creation. Protein and homology models were generated using Prime ${ }^{18}$ (Schrödinger Release 2019-3: Prime, Schrödinger, LLC, New York, NY, 2020). For protein models, crystal structures were imported into Prime ${ }^{18}$ by PDB
ID (1FUU, 2J0S, 5ZC9). Using Protein Preparation Wizard, missing loops and side chains were filled in, missing hydrogens were added, and the protein underwent restrained energy minimization. Water molecules with less than three contacts with the protein were removed. For homology modeling, the amino acid sequence was obtained from Uniprot ${ }^{19}$ and using multiple sequence alignment, proteins with high degrees of homology were selected as structural templates (PDB: 1FUU, 2J0S; Supporting Information Figure 6). All homology models underwent loop refinement using serial loop sampling. For all models, molecular dynamic simulations were run to assess the model quality and stability. For models with cocrystallized ligands (2J0s and 5ZC9), ligands were manually removed and then redocked into the binding site to assess the model accuracy in predicted experimentally derived binding pose.

Docking Grids. Receptor docking grids were generated for virtual screening with Glide ${ }^{32}$ Receptor Grid Generation (Schrödinger Release 2019-3: Glide, Schrödinger, LLC, New York, NY, 2019) and OpenEye's Make_Receptor function ${ }^{25}$ (OpenEye Scientific Software, Santa Fe, NM, http://www. eyesopen.com. OEDOCKING 3.3.0). All proteins were initially prepared using Protein Prep Wizard ${ }^{18}$ prior to grid generation. Grids were then generated using the cocrystallized ligand as the centroid for docking similarly sized ligands (protein structures 5ZC9 and 2J0S). For the 1FUU model for which no ligand was cocrystallized, docking grids were made using the centroid of known residues, which define the ATPbinding site. Docking restrictions were made in the grid to ensure certain hydrogen-bond interactions were maintained during docking, particularly nucleotide interaction of conserved glutamines in the N-terminal domain and motif IV arginines in the C-terminal of eIF4A1. For large-scale docking studies, docking grids were generated using the same above methods using protein structures exported from clustered trajectory frames of molecular dynamics simulations.

Molecular Dynamics (MD). All-atom explicit solvent molecular dynamics (MD) simulations were run using the Desmond $^{33}$ GPU accelerated suite of Schrödinger (Schrödinger Release 2019-1 Desmond Molecular Dynamics System, D. E. Shaw Research, New York, NY, 2020; Maestro-Desmond Interoperability Tools, Schrödinger, New York, NY, 2020). Simulation times varied between $300 \mathrm{~ns}$ and $3 \mathrm{~ms}$, with a maximum of 3000 frames and a recording interval of $1 / 1000$ th of the overall simulation time. NPT (isothermal-isobaric) ensemble was used for the simulation, which most accurately represents laboratory conditions at ambient temperature and pressure $(300 \mathrm{~K}$ and $1.01325 \mathrm{bar})$. Each model system was relaxed before the simulation for several nanoseconds, which explains the slight variability in initial interdomain linker distance measured in these experiments. Systems were built also using Desmond with the SPC solvent model and OPLS3e forcefield. Optimized potentials for liquid simulations (OPLS), developed by Jorgensen et al., ${ }^{34}$ is a forcefield with a core set of nonbonded parameters. OPLS3 is the latest model, which builds on optimization of valence and torsion parameters, parametrization of bond-charge correction terms, and includes machine learning data from quantum chemical simulations to improve the representation of secondary structure elements in proteins and protein-ligand binding. Simulations were computed across dedicated GPU cores.

Ligand Library Preparation. Compounds selected for virtual screen were populated as SMILES in a commaseparated file along with a unique identifier or catalog number 
from the vendor. Using Pipeline Pilot (BIOVIA Pipeline Pilot, Release 2018, San Diego: Dassault Systèmes), SMILES were canonicalized and the file was transformed to a structure data file (SDF) to be used as input for LigPrep (Schrödinger Release 2019-3: LigPrep, Schrödinger, LLC, New York, NY, 2019) or for OpenEye QUACPAC 2.0.1 and OMEGA 3.1.1 (OpenEye Scientific Software, Santa Fe, NM, http://www. eyesopen.com). For initial large-scale virtual screens with over 100000 compounds, OpenEye Hybrid ${ }^{25}$ docking was utilized. Following enrichment by Hybrid Docking Score, smaller subsets of ligands were then used to dock using Glide (Schrödinger) for further binding pose visualization and MD simulations. Ligand libraries were obtained from Enamine's Advanced HTS library, MolportDB, and eMolecules (2019 Release). The eMolecule library was filtered into screening subsets using both lead-like and drug-like filters provided by OpenEye. For ligand preparation, default settings were used in both programs. Compounds to be used in OpenEye were prepared first using QUACPAC, which involves tautomer and protomer enumeration. OMEGA was then used to generate up to 150 conformers for each ligand. For GLIDE docking, ligands were prepared using LigPrep (Schrödinger Release 2019-3: LigPrep, Schrödinger, LLC, New York, NY, 2021), which generates all conformers, tautomers, and ionization states.

Ligand Shape Screen. Shape screens were conducted using fastROCS GPU ${ }^{23}$ 3.3.0 (OpenEye Scientific Software, Santa Fe, NM, http://www.eyesopen.com), which can screen millions of compounds in seconds. A combined Tanimoto shape and color score was used to filter the most similar compounds in eMolecules, MolportDB, and Enamine-prepared compound libraries. Shape screen was used to first identify ATP/nucleotide mimetics and was further used to find analogues with the piperazine scaffold of compounds UM139 and UM127.

Ligand Docking. For initial large-scale docking studies, OpenEye's HYBRID Dock ${ }^{25}$ 3.3.1 was used. HYBRID docking was done in parallel on all eIF4A-I grids in one job. The top 25000 compounds (by docking score) were exported for final analysis. For smaller-scale docking jobs or confirmatory docking studies prior to the MD simulation, Glide Docking ${ }^{32}$ was used (Schrödinger Release 2019-3: Glide, Schrödinger, LLC, New York, NY, 2019). All default settings were used. Glide standard precision docking (SP) was performed using 3D ligands from LigPrep. Up to 25 poses were generated per ligand with the top five poses being selected for output. Glide scores were exported for aggregation across various grids using Pipeline Pilot. GLIDE docking scoring has been described extensively by Friesner et al. ${ }^{35}$ HYBRID docking scoring is described by McGann. ${ }^{25}$

Analysis. Molecular dynamic analyses of eIF4A1 models with key ligands were conducted using Desmond Simulation Event Analyses, which included RMSD, RMSF, and ligandprotein interactions. Compounds were analyzed using QikProp for predicted physiochemical properties. Compounds were clustered by scaffold similarity using Pipeline Pilot with extended fingerprint methods. Energies of each ligand-protein system were calculated using Prime ${ }^{18}$ MMGBSA (Schrödinger Release 2019-1: Prime, Schrödinger, LLC, New York, NY, 2019).

Compound Storage and Cataloging. All compounds were purchased as powders in quantities less than $5 \mathrm{mg}$. Compounds were dissolved in various organic solvents (methanol, dichloromethane, ethyl acetate) and were aliquoted out in separate vials. Solvents were evaporated using a rotovap and high vacuum and aliquoted powders were stored at -20 ${ }^{\circ} \mathrm{C}$. Compounds were dissolved in DMSO to a concentration of $10 \mathrm{mM}$ prior to use in assays. Compounds were stored in DMSO at $-80{ }^{\circ} \mathrm{C}$ no longer than 1 week. All purchased compounds were cataloged virtually using the Collaborative Drug Discovery (CDD) Vault (https://www. collaborativedrug.com/).

elF4A1 Inhibitor ATPase Assay. Recombinant eIF4A was added to a clear-bottom 384 well plate (Greiner) in ATPase buffer $\left(20 \mathrm{mM}\right.$ Tris $\mathrm{pH} 7.4,80 \mathrm{mM} \mathrm{KCl}, 2.5 \mathrm{mM} \mathrm{MgCl}_{2}, 1$ $\mathrm{mM} \mathrm{DTT}, 1 \%$ glycerol). Compounds were added and the plate at concentrations starting at $250 \mu \mathrm{M}$ and were incubated at 37 ${ }^{\circ} \mathrm{C}$ for $20 \mathrm{~min}$. ATP/RNA solution was added to afford a 20 $\mu \mathrm{L}$ per well reaction volume with $750 \mathrm{nM}$ eIF4A, $250 \mu \mathrm{M}$ ATP, and $0.25 \mathrm{mg} / \mathrm{mL}$ yeast RNA (Sigma). The plate was incubated for $4 \mathrm{~h}$ at $37^{\circ} \mathrm{C}$. Tween-20 $(0.04 \% \mathrm{v} / \mathrm{v})$ was added to a Malachite Green solution, and then it was added to wells (40 $\mu \mathrm{L}$ per well). The solution was incubated for $5 \mathrm{~min}$ at room temperature and A660 was read on a SpectraMax iD5 plate reader (Molecular Devices). Experiments were conducted in triplicate. $\mathrm{IC}_{50}$ values were calculated using nonlinear regression fit in Graphpad Prism7.

elF4A1 Cell-Based Viability Screen. Cells were plated in triplicate at $3-5 \times 10^{3}$ cells/well in serial dilutions of drug ranging two logs with the top concentration for $1 \mu \mathrm{M}$ silvestrol. Viability was measured after $72 \mathrm{~h}$ using Cell Titer Glo (Promega G7573) following manufacturer's protocol. Luminescence was detected on the BioTek HT Synergy plate reader and $\mathrm{LD}_{50}$ values calculated using nonlinear regression fit in Graphpad Prism7.

\section{ASSOCIATED CONTENT}

\section{Supporting Information}

The Supporting Information is available free of charge at https://pubs.acs.org/doi/10.1021/acsomega.1c02805.

1FUU homology model MD analysis (Figure S1); interdomain distances of the native 1FUU model (Figure S2); interdomain analysis of 2J0S molecular dynamic simulation (Figure S3); cell-viability data for eIF4A1 inhibitors (Figure S4); binding hypothesis from molecular dynamics of UM162 (Figure S5); sequence alignment of eIF4A1 for homology modeling (Figure S6) (PDF)

eIF4A1 compound activity data (Table S1) (XLSX)

\section{AUTHOR INFORMATION}

\section{Corresponding Author}

Stephan C. Schürer - Department of Molecular and Cellular Pharmacology, Miller School of Medicine, University of Miami, Miami, Florida 33136, United States; Sylvester Comprehensive Cancer Center, University of Miami Health System, Miami, Florida 33136, United States; Institute for Data Science \& Computing, University of Miami, Miami, Florida 33136, United States; ○ orcid.org/0000-0001-

7180-0978; Email: sschurer@miami.edu

\section{Authors}

Derek J. Essegian - Department of Molecular and Cellular Pharmacology, Miller School of Medicine, University of Miami, Miami, Florida 33136, United States; Medical 
Scientist Training Program, Miller School of Medicine, University of Miami, Miami, Florida 33136, United States

Tyler A. Cunningham - Department of Molecular and Cellular Pharmacology, Miller School of Medicine, University of Miami, Miami, Florida 33136, United States; Medical Scientist Training Program, Miller School of Medicine, University of Miami, Miami, Florida 33136, United States

Christopher J. Zerio - Department of Pharmacology and Toxicology, College of Pharmacy, The University of Arizona, Tuscon, Arizona 85721, United States; (1) orcid.org/00000003-4053-4835

Eli Chapman - Department of Pharmacology and Toxicology, College of Pharmacy, The University of Arizona, Tuscon, Arizona 85721, United States; orcid.org/0000-00026310-1664

Jonathan Schatz - Sylvester Comprehensive Cancer Center, University of Miami Health System, Miami, Florida 33136, United States; Department of Medicine, Miller School of Medicine, University of Miami, Miami, Florida 33136, United States

Complete contact information is available at: https://pubs.acs.org/10.1021/acsomega.1c02805

\section{Notes}

The authors declare no competing financial interest.

\section{ACKNOWLEDGMENTS}

This work was partially supported by NIH grants P30CA240139 (Cancer Center Support Grant), U54HL127624 (BD2K-LINCS DCIC), and U24TR002278 (IDG RDOC). The authors thank ChemAxon for providing the academic research license for their Cheminformatics software tools including JChem for Excel and the Marvin tools. The authors also thank D. E. Shaw Research and Schrödinger for the academic license to the molecular dynamics (MD) simulation package and OpenEye Scientific Software for their academic research licenses. The authors acknowledge resources from Sylvester Comprehensive Cancer Center and the Institute for Data Science \& Computing at the University of Miami.

\section{REFERENCES}

(1) Raza, F.; Waldron, J. A.; Quesne, J. L. Translational dysregulation in cancer: eIF4A isoforms and sequence determinants of eIF4A dependence. Biochem. Soc. Trans. 2015, 43, 1227-1233.

(2) Pelletier, J.; Graff, J.; Ruggero, D.; Sonenberg, N. Targeting the eIF4F translation initiation complex: a critical nexus for cancer development. Cancer Res. 2015, 75, 250-263.

(3) Shahbazian, D.; Roux, P. P.; Mieulet, V.; Cohen, M. S.; Raught, B.; Taunton, J.; Hershey, J. W.; Blenis, J.; Pende, M.; Sonenberg, N. The mTOR/PI3K and MAPK pathways converge on eIF4B to control its phosphorylation and activity. EMBO J. 2006, 25, 27812791.

(4) Chan, K.; Robert, F.; Oertlin, C.; Kapeller-Libermann, D.; Avizonis, D.; Gutierrez, J.; Handly-Santana, A.; Doubrovin, M.; Park, J.; Schoepfer, C.; Da Silva, B.; Yao, M.; Gorton, F.; Shi, J.; Thomas, C. J.; Brown, L. E.; Porco, J. A., Jr.; Pollak, M.; Larsson, O.; Pelletier, J.; Chio, I. I. C. eIF4A supports an oncogenic translation program in pancreatic ductal adenocarcinoma. Nat. Commun. 2019, 10, No. 5151.

(5) Modelska, A.; Turro, E.; Russell, R.; Beaton, J.; Sbarrato, T.; Spriggs, K.; Miller, J.; Graf, S.; Provenzano, E.; Blows, F.; Pharoah, P.; Caldas, C.; Le Quesne, J. The malignant phenotype in breast cancer is driven by eIF4A1-mediated changes in the translational landscape. Cell Death Dis. 2015, 6, e1603.
(6) Zhong, X.; Persaud, L.; Muharam, H.; Francis, A.; Das, D.; Aktas, B. H.; Sauane, M. Eukaryotic Translation Initiation Factor 4A Down-Regulation Mediates Interleukin-24-Induced Apoptosis through Inhibition of Translation. Cancers 2018, 10, No. 153.

(7) Peters, T. L.; Tillotson, J.; Yeomans, A. M.; Wilmore, S.; Lemm, E.; Jimenez-Romero, C.; Amador, L. A.; Li, L.; Amin, A. D.; Pongtornpipat, P.; Zerio, C. J.; Ambrose, A. J.; Paine-Murrieta, G.; Greninger, P.; Vega, F.; Benes, C. H.; Packham, G.; Rodriguez, A. D.; Chapman, E.; Schatz, J. H. Target-Based Screening against eIF4A1 Reveals the Marine Natural Product Elatol as a Novel Inhibitor of Translation Initiation with In Vivo Antitumor Activity. Clin. Cancer Res. 2018, 24, 4256-4270.

(8) Bordeleau, M.-E.; Robert, F.; Gerard, B.; Lindqvist, L.; Chen, S. M.; Wendel, H. G.; Brem, B.; Greger, H.; Lowe, S. W.; Porco, J. A., Jr.; Pelletier, J. Therapeutic suppression of translation initiation modulates chemosensitivity in a mouse lymphoma model. J. Clin. Invest. 2008, 118, 2651-2660.

(9) Tillotson, J.; Kedzior, M.; Guimaraes, L.; Ross, A. B.; Peters, T. L.; Ambrose, A. J.; Schmidlin, C. J.; Zhang, D. D.; Costa-Lotufo, L. V.; Rodriguez, A. D.; Schatz, J. H.; Chapman, E. ATP-competitive, marine derived natural products that target the DEAD box helicase, eIF4A. Bioorg. Med. Chem. Lett. 2017, 27, 4082-4085.

(10) Gupta, S. V.; Sass, E. J.; Davis, M. E.; Edwards, R. B.; Lozanski, G.; Heerema, N. A.; Lehman, A.; Zhang, X.; Jarjoura, D.; Byrd, J. C.; Pan, L.; Chan, K. K.; Kinghorn, A. D.; Phelps, M. A.; Grever, M. R.; Lucas, D. M. Resistance to the translation initiation inhibitor silvestrol is mediated by $\mathrm{ABCB} 1 / \mathrm{P}$-glycoprotein overexpression in acute lymphoblastic leukemia cells. AAPS J. 2011, 13, 357-364.

(11) Iwasaki, S.; Iwasaki, W.; Takahashi, M.; Sakamoto, A.; Watanabe, C.; Shichino, Y.; Floor, S. N.; Fujiwara, K.; Mito, M.; Dodo, K.; Sodeoka, M.; Imataka, H.; Honma, T.; Fukuzawa, K.; Ito, T.; Ingolia, N. T. The Translation Inhibitor Rocaglamide Targets a Bimolecular Cavity between eIF4A and Polypurine RNA. Mol. Cell 2019, 73, 738-748.

(12) Caruthers, J. M.; Johnson, E. R.; McKay, D. B. Crystal structure of yeast initiation factor 4A, a DEAD-box RNA helicase. Proc. Natl. Acad. Sci. U.S.A. 2000, 97, 13080-13085.

(13) Andreou, A. Z.; Klostermeier, D. The DEAD-box helicase eIF4A: paradigm or the odd one out? RNA Biol. 2013, 10, 19-32.

(14) Linder, P.; Jankowsky, E. From unwinding to clamping - the DEAD box RNA helicase family. Nat. Rev. Mol. Cell Biol. 2011, 12, 505-516.

(15) Shen, L.; Pelletier, J. Selective targeting of the DEAD-box RNA helicase eukaryotic initiation factor (eIF) $4 \mathrm{~A}$ by natural products. Nat. Prod. Rep. 2020, 37, 609-616.

(16) Marintchev, A.; Edmonds, K. A.; Marintcheva, B.; Hendrickson, E.; Oberer, M.; Suzuki, C.; Herdy, B.; Sonenberg, N.; Wagner, G. Topology and regulation of the human eIF4A/4G/4H helicase complex in translation initiation. Cell 2009, 136, 447-460.

(17) Altschul, S. F.; Gish, W.; Miller, W.; Myers, E. W.; Lipman, D. J. Basic local alignment search tool. J. Mol. Biol. 1990, 215, 403-410.

(18) Jacobson, M. P.; Pincus, D. L.; Rapp, C. S.; Day, T. J.; Honig, B.; Shaw, D. E.; Friesner, R. A. A hierarchical approach to all-atom protein loop prediction. Proteins 2004, 55, 351-367.

(19) Bateman, A.; The UniProt Consortium; et al. UniProt: the universal protein knowledgebase in 2021. Nucleic Acids Res. 2021, 49, D480-D489.

(20) Meng, H.; Li, C.; Wang, Y.; Chen, G. Molecular dynamics simulation of the allosteric regulation of eIF4A protein from the open to closed state, induced by ATP and RNA substrates. PLoS One 2014, 9, No. e86104.

(21) Bono, F.; Ebert, J.; Lorentzen, E.; Conti, E. The crystal structure of the exon junction complex reveals how it maintains a stable grip on mRNA. Cell 2006, 126, 713-725.

(22) Hawkins, P. C. D.; Nicholls, A. Conformer Generation with OMEGA: Learning from the Data Set and the Analysis of Failures. J. Chem. Inf. Model. 2012, 52, 2919-2936. 
(23) Hawkins, P. C. D.; Skillman, A. G.; Nicholls, A. Comparison of shape-matching and docking as virtual screening tools. J. Med. Chem. 2007, 50, 74-82.

(24) Bajusz, D.; Racz, A.; Heberger, K. Why is Tanimoto index an appropriate choice for fingerprint-based similarity calculations? J. Cheminformatics 2015, 7, No. 20.

(25) McGann, M. FRED and HYBRID docking performance on standardized datasets. J. Comput. Aided Mol. Des. 2012, 26, 897-906.

(26) Tang, G.-Y. Why Polyphenols have Promiscuous Actions? An Investigation by Chemical Bioinformatics. Nat. Prod. Commun. 2016, 11, 655-656.

(27) Barrajón-Catalán, E.; Herranz-Lopez, M.; Joven, J.; SeguraCarretero, A.; Alonso-Villaverde, C.; Menendez, J. A.; Micol, V. Molecular promiscuity of plant polyphenols in the management of age-related diseases: far beyond their antioxidant properties. Adv. Exp. Med. Biol. 2014, 824, 141-159.

(28) Ernst, J. T.; Thompson, P. A.; Nilewski, C.; Sprengeler, P. A.; Sperry, S.; Packard, G.; Michels, T.; Xiang, A.; Tran, C.; Wegerski, C. J.; Eam, B.; Young, N. P.; Fish, S.; Chen, J.; Howard, H.; Staunton, J.; Molter, J.; Clarine, J.; Nevarez, A.; Chiang, G. G.; Appleman, J. R.; Webster, K. R; Reich, S. H. Design of Development Candidate eFT226, a First in Class Inhibitor of Eukaryotic Initiation Factor 4A RNA Helicase. J. Med. Chem. 2020, 63, 5879-5955.

(29) Taroncher-Oldenburg, G.; Muller, C.; Obermann, W.; Ziebuhr, J.; Hartmann, R. K.; Grunweller, A. Targeting the DEAD-Box RNA Helicase eIF4A with Rocaglates-A Pan-Antiviral Strategy for Minimizing the Impact of Future RNA Virus Pandemics. Microorganisms 2021, 9, No. 540.

(30) Bathula, R.; Lanka, G.; Muddagoni, N.; Dasari, M.; Nakkala, S.; Bhargavi, M.; Somadi, G.; Sivan, S. K.; Rajender Potlapally, S. Identification of potential Aurora kinase-C protein inhibitors: an amalgamation of energy minimization, virtual screening, prime MMGBSA and AutoDock. J. Biomol. Struct. Dyn. 2020, 38, 23142325.

(31) Singh, T.; Adekoya, O. A.; Jayaram, B. Understanding the binding of inhibitors of matrix metalloproteinases by molecular docking, quantum mechanical calculations, molecular dynamics simulations, and a MMGBSA/MMBappl study. Mol. BioSyst. 2015, 11, 1041-1051.

(32) Halgren, T. A.; Murphy, R. B.; Friesner, R. A.; Beard, H. S.; Frye, L. L.; Pollard, W. T.; Banks, J. L. Glide: A new approach for rapid, accurate docking and scoring. 2. Enrichment factors in database screening. J. Med. Chem. 2004, 47, 1750-1759.

(33) Jiang, W.; Phillips, J. C.; Huang, L.; Fajer, M.; Meng, Y.; Gumbart, J. C.; Luo, Y.; Schulten, K.; Roux, B. Generalized Scalable Multiple Copy Algorithms for Molecular Dynamics Simulations in NAMD. Comput. Phys. Commun. 2014, 185, 908-916.

(34) Jorgensen, W. L.; Tirado-Rives, J. The OPLS [optimized potentials for liquid simulations] potential functions for proteins, energy minimizations for crystals of cyclic peptides and crambin. J. Am. Chem. Soc. 1988, 110, 1657-1666.

(35) Friesner, R. A.; Banks, J. L.; Murphy, R. B.; Halgren, T. A.; Klicic, J. J.; Mainz, D. T.; Repasky, M. P.; Knoll, E. H.; Shelley, M.; Perry, J. K.; Shaw, D. E.; Francis, P.; Shenkin, P. S. Glide: a new approach for rapid, accurate docking and scoring. 1. Method and assessment of docking accuracy. J. Med. Chem. 2004, 47, 1739-1749. 